\title{
Monochorionic-Triamniotic Triplet Pregnancy Complicated by Twin Reversed Arterial Perfusion Sequence: Case Report and Literature Review
}

\author{
Pingshan Pan, MD ${ }^{1}$ Guoyang Luo, MD, PhD ${ }^{2}$ Lu Tang, BD ${ }^{1}$ Jeanne D. Rolle, BS ${ }^{2}$ Yuqin Qin, MD ${ }^{1}$ \\ Quan Zeng, $\mathrm{BD}^{1}$ jiangting Wei, $\mathrm{MD}^{1}$ Yuanfang Chen, MD ${ }^{1}$ Hongwei Wei, MD ${ }^{1}$ \\ ${ }^{1}$ Department of Obstetrics, Guangxi Zhuang Autonomous Region \\ Women and Children Health Care Hospital, Nanning, Guangxi, \\ People's Republic of China \\ 2 Department of Obstetrics and Gynecology, University of \\ Connecticut, Farmington, Connecticut

\begin{abstract}
Address for correspondence Hongwei Wei, MD, Department of Care Hospital No.59, Xiangzhu Road, Nanning City, Guangxi Province,
\end{abstract} \\ Obstetrics, Guangxi Zhuang Autonomous Region Women and Children \\ 530012, People's Republic of China (e-mail: 35743650@qq.com).
}

Am J Perinatol Rep 2017;7:e106-e110.

\begin{abstract}
Background Monochorionic-triamniotic pregnancies are rare and fraught with complications.

Case A case of monochorionic-triamniotic triplet pregnancy complicated by twin reversed arterial perfusion (TRAP) sequence is presented. The patient declined termination or selective fetal reduction. Triplet C was acardiac. At 24 weeks, triplet B developed polyhydramnios. At 30 weeks, polyhydramnios was seen in all three amniotic sacs, but without signs of fetal hydrops and with normal Doppler velocimetry measurements in the umbilical artery, middle cerebral artery, and ductus arteriosus of triplets $A$ and B. At $32^{2 / 7}$ weeks, the patient presented with preterm premature rupture of membranes and preterm labor. Two live male infants were delivered by cesarean delivery weighing 1,350 and

Keywords

- monochorionic

- triplet pregnancy

- acardiac fetus

- twin-reversed arterial perfusion sequence $1,390 \mathrm{~g}$, respectively; the acardiac fetus weighed $1,460 \mathrm{~g}$. Pathology examination revealed a single placenta weighing $1,250 \mathrm{~g}$, with evidence of direct vascular connections between triplets $\mathrm{A}$ and $\mathrm{C}$ as well as between triplets $\mathrm{A}$ and $\mathrm{B}$.

Conclusion Monochorionic-triamniotic triplet pregnancy with TRAP sequence is rare. Although the risk of complications is high, such pregnancies can be managed conservatively in select cases.
\end{abstract}

Spontaneous triplet pregnancies are rare, with a prevalence of approximately 1 in 7,000 deliveries. However, with the increasing availability of assisted reproductive technologies, the rate of high-order multiple pregnancies (triplets and above) has risen dramatically over the last 20 years. ${ }^{1}$ Triplet pregnancies are at a high risk of pregnancy complications and have higher perinatal morbidity and mortality rates, such as structural defects, and neurodevelopmental anomalies. Monochorionic-triplet pregnancies are exceedingly rare, with an estimated prevalence of 1 in 100,000 births. ${ }^{2,3}$ TRAP sequence is a unique complication of monozygotic pregnancies, and with

received

February 21, 2017

accepted after revision

April 21, 2017
DOI https://doi.org/

10.1055/s-0037-1603917.

ISSN 2157-6998. a reported incidence of 1 in 35,000 twin gestations. ${ }^{4}$ The true incidence of TRAP sequence in trichorionic triplet gestation is not known. Here, we report a case of a monochorionic-triamniotic triplet pregnancy complicated by TRAP sequence along with a review of the published literature.

\section{Case Report}

A 25-year-old woman, gravida 4, para 2, was referred to our perinatal center at 17 weeks of gestation with a suspected triplet gestation. Ultrasound examination revealed a
Copyright $\odot 2017$ by Thieme Medical Publishers, Inc., 333 Seventh Avenue, New York, NY 10001, USA. Tel: +1(212) 584-4662.
License terms

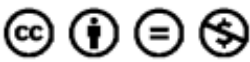




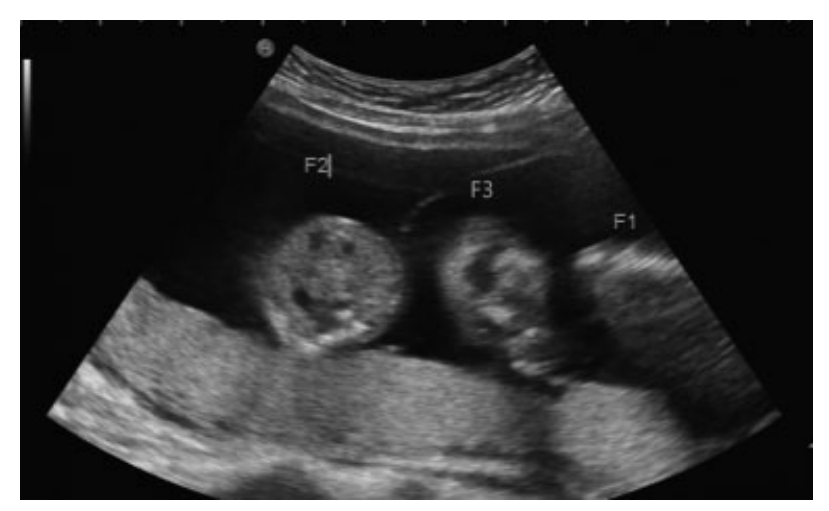

Fig. 1 Ultrasound images of a monochorionic-triamniotic triplet pregnancy at 17 weeks. The acardiac fetus is seen in the middle of the screen (F3). F1, fetus one; F2, fetus two; F3, fetus three.

monochorionic-triamniotic triplet gestation with two structurally normal fetuses and an acardiac triplet (-Fig. 1). Detailed anatomy scan and fetal echocardiogram did not detect any structural abnormalities in triplets A and B. After extensive counseling; the patient declined pregnancy termination or selective fetal reduction of the acardiac fetus. The pregnancy was followed with ultrasound examinations every 2 weeks. At 24 weeks, triplet B developed polyhydramnios with an amniotic fluid maximal vertical pocket (MVP) of $11.9 \mathrm{~cm}$; triplet A had normal amniotic fluid (MVP: $7.3 \mathrm{~cm}$ ). Triplet $\mathrm{C}$ (the acardiac fetus) showed diffuse subcutaneous edema. By 30 weeks, polyhydramnios was seen in all three fetuses with MVPs of $8.1 \mathrm{~cm}$ (fetus A), $10.5 \mathrm{~cm}$ (fetus B), and $12.5 \mathrm{~cm}$ (fetus C, acardiac), respectively (-Fig. 2). Doppler velocimetry of the umbilical artery, middle cerebral artery, and ductus arteriosus were normal for triplets A and $B$, and there was no evidence of fetal hydrops. The patient received an empiric course of antenatal corticosteroids for induction of fetal lung maturation at 30 weeks.

At $32^{2 / 7}$ weeks, the patient presented with preterm premature rupture of membranes and unstoppable preterm labor. A cesarean delivery was performed, and two liveborn male infants were delivered. Triplet A weighed 1,350 g with Apgar scores of 7 and 9 at 1 and 5 minutes, respectively. Triplet B weighed $1,390 \mathrm{~g}$ with Apgar scores of 7 and 9 at 1 and 5 minutes, respectively. The acardiac fetus (triplet $\mathrm{C}$ ) weighed $1,460 \mathrm{~g}$ with significant tissue edema. The patient had an uncomplicated postpartum course. At 6 months of age, both children showed normal growth and neurodevelopment.
Placental pathology revealed a single placenta weighing $1,250 \mathrm{~g}$. The placental cord insertion of triplets $A$ and $C$ (acardiac) were only $0.5 \mathrm{~cm}$ apart (-Fig. 3A). Superficial vascular connections were identified between triplets $A$ and C as well as between triplets A and B (-Fig. 3B).

\section{Discussion}

Monochorionic-triplet pregnancies are exceedingly rare with a reported incidence of 1 in 100,000 births. ${ }^{2,3}$ Monozygotic multiple gestations occur when a zygote splits into two or more genetically identical embryos. The timing of this division determines the chorionicity (i.e., the configuration of the placentas and fetal membranes). The later the division, the greater the likelihood of shared tissue elements among conceptuses. Chorionicity is a major determinant of pregnancy outcome and thus dictates obstetric management. It is best determined by ultrasound in the first trimester.

TRAP sequence is a serious complication that occurs exclusively in monochorionic pregnancies. It is caused by one or more abnormal vascular connections between fetuses that occur most commonly on the surface of the placenta or, in some cases, within the umbilical cord. As a result, instead of diving down into the placenta, the blood returning from one fetus (the donor or "pump fetus") goes directly to the second fetus (the recipient) and then back to the placenta. As a result, the recipient twin receives blood that is low in oxygen and nutrient content, and that is traveling in the wrong direction through its arterial circulation; hence the term, TRAP sequence. While there may be enough residual oxygen and nutrients to support the development of the recipient fetus's lower extremities, the upper extremities, and head typically do not develop. Similarly, since the blood is circulating in the reverse direction, the heart is usually a rudimentary, nonfunctional single-chambered organ (termed "acardiac"). ${ }^{4-6}$ The acardiac fetus will not survive. Survival of the donor (pump) fetus(es) is dependent on the ability of its heart to perfuse not only its own body but also of its acardiac sibling. Heart failure is common, with an overall risk of the intrauterine fetal demise of over $50 \%$. 7,8

TRAP sequence is a sonographic diagnosis. Findings include identification of a monochorionic multiple pregnancies in which one fetus has severe malformations, usually lacking a functional heart and a cephalic pole. Given its unusual shape and size, it has also been called the "acardiac acephalus" or

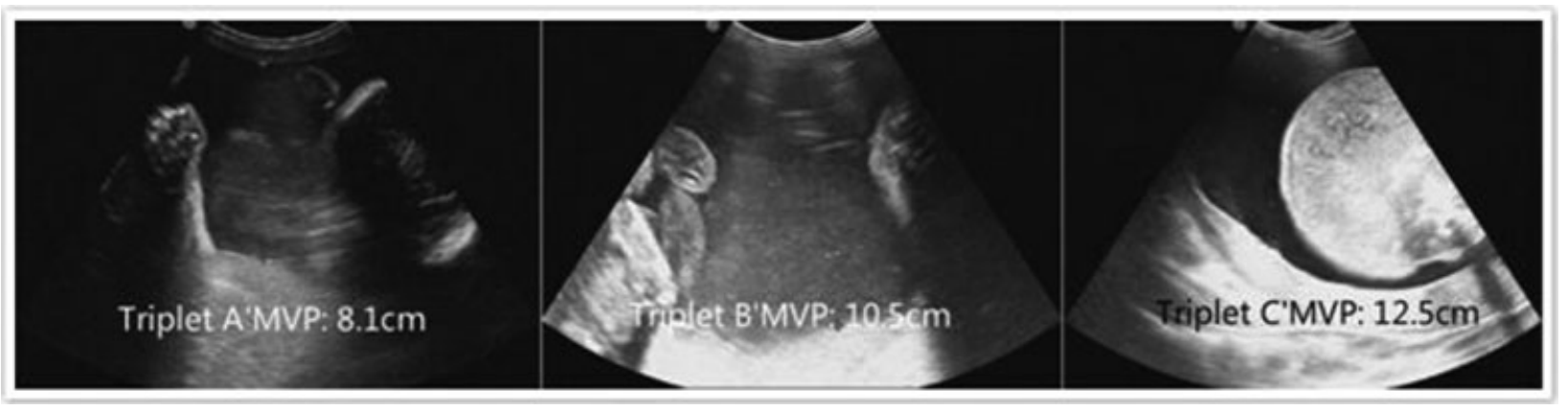

Fig. 2 Polyhydramnios was present in all three fetuses by 30 weeks. MVP, maximal vertical pocket. 


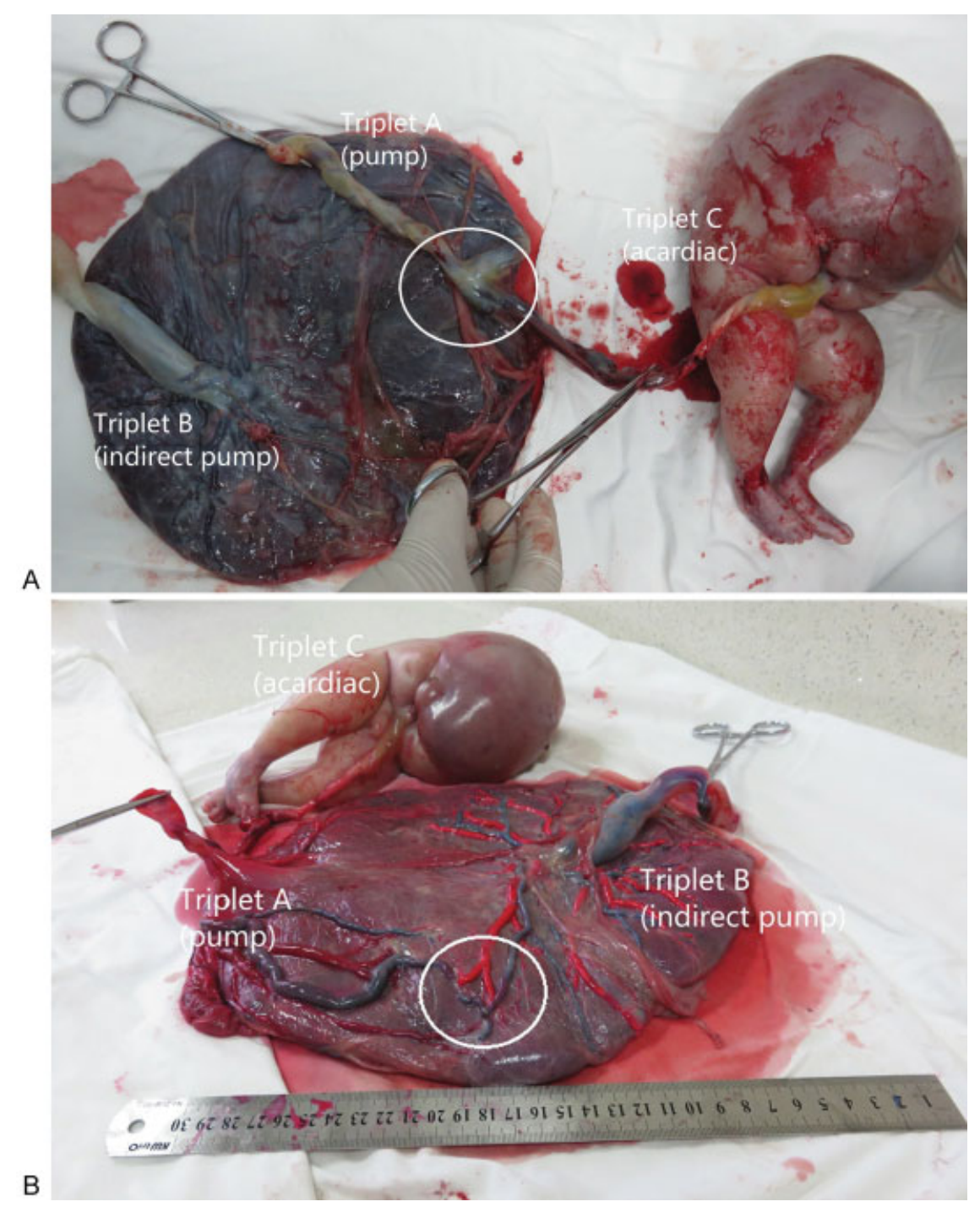

Fig. 3 (A and B) Placenta surface anastomoses among the three fetuses after delivery.

"acardiac monster." Doppler velocimetry will confirm reversed arterial perfusion in the acardiac twin. It may also be able to identify and characterize the abnormal vascular communications within the placenta or umbilical cord. The incidence of TRAP sequence is estimated as 1 in 35,000 pregnancies, but increases to 1 in 100 monochorionic twin gestations, and 1 in 30 triplet pregnancies. Given that the low prevalence of monochorionic-triamniotic triplet pregnancy, the incidence of TRAP sequence in monochorionic-triplet pregnancy is exceedingly rare. On review of the published literature, we identified 11 case reports, which are summarized in - Table 1 .

The hemodynamic changes in a monochorionic-triplet pregnancy with TRAP sequence are even more complicated since it involves three fetuses. The abnormal vascular anastomoses could exist between two fetuses or among all three fetuses. When two normally developed fetuses and one acardiac fetus coexist, the "pump" could be driven by only one of the normal fetuses or by both fetuses. Among the six prior reported cases, we identified three types of pumps: (1) one in which both normal fetuses pump blood to the acardiac triplet (i.e., two direct pump fetuses); (2) one in which one fetus pumps blood to the acardiac triplet, and the other is unaffected (i.e., one direct pump fetus); and (3) one in which there is one direct and one indirect pump fetus (i.e., one structurally normal triplets pumps blood to the other structurally normal fetus, which then sends the blood on to the acardiac triplet). In our case, placental pathology showed direct arterial-to-arterial anastomoses between triplet $B$ (the indirect pump fetus) and triplet $A$ (the direct pump fetus) as well as between triplet $A$ and triplet $C$ (the acardiac fetus); no vascular anastomoses were identified between triplets B and C. As such, triplet A served as the direct pump and $B$ as an indirect pump fetus; it is as though the pumps are in series rather than in parallel. Although polyhydramnios developed in all three amniotic sacs, fetuses A and B had a normal outcome with no significant growth discordance or fetal hydrops.

The prognosis of the pump fetus(es) is closely related to the presence or absence of hemodynamic complications. Early diagnosis and careful counseling are crucial to optimize management. Options include pregnancy termination or selective fetal reduction. The selective reduction can be performed with radiofrequency ablation of the aorta of the acardiac twin or 


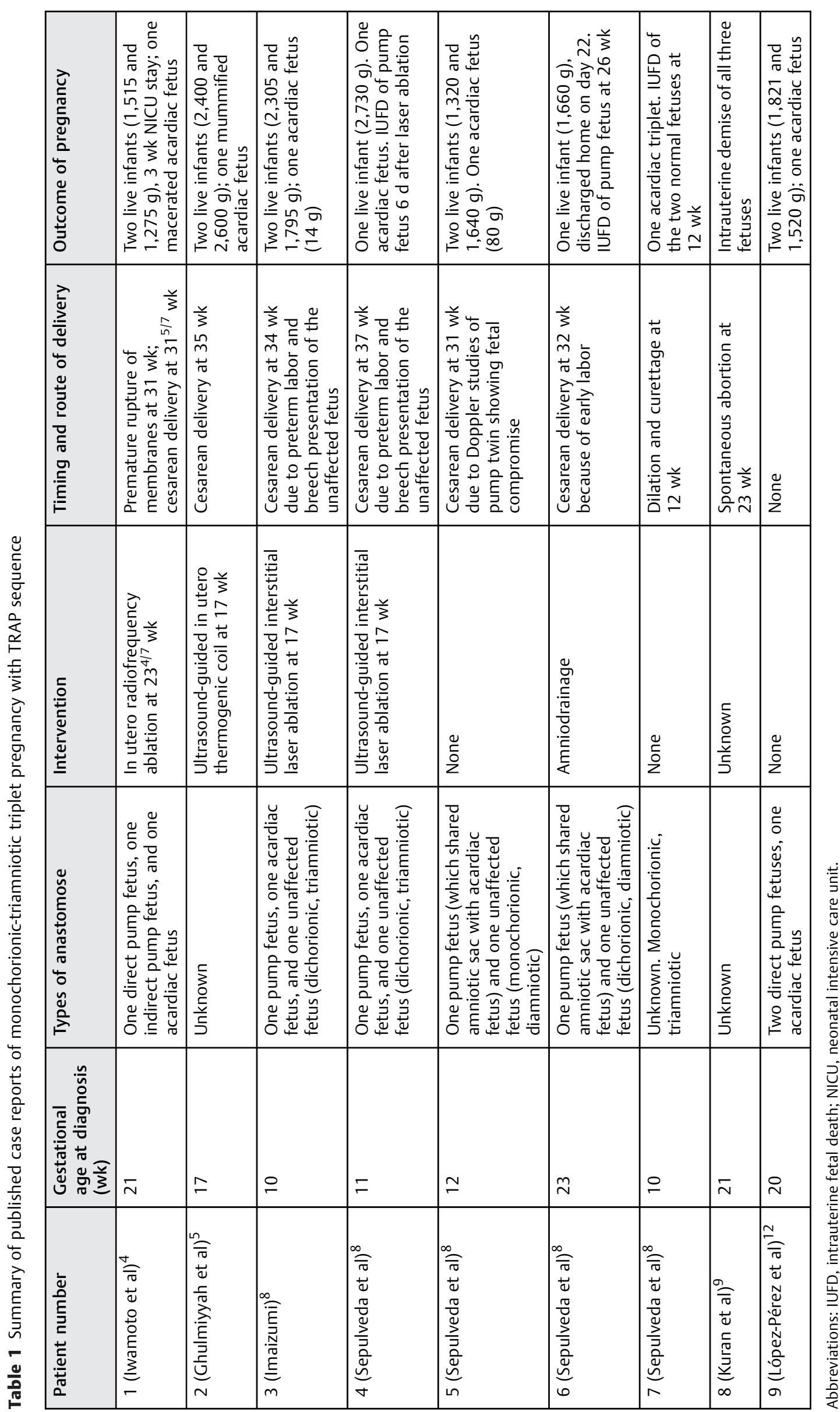


fetoscopic cord ligation. Although minimally invasive, such treatment can prolong time to delivery and improve survival rates up to 70 to $95 \% .^{7,8}$ Such pregnancies should be followed with frequency ultrasound monitoring. When early delivery is anticipated, antenatal corticosteroids should be considered to promote fetal lung maturity. With expectant management, the survival rate of the pump fetus is only around $50 \%{ }^{8,9}$ Prognosis is also closely related to the size of the acardiac fetus. ${ }^{10}$ Indications for active intervention in multiple pregnancies with TRAP sequence include: (1) if the abdominal circumference of the acardiac fetus is equivalent to or bigger than that of the pump fetus; (2) polyhydramnios (MVP > $8 \mathrm{~cm}$ ); (3) severe blood flow abnormality in the pump fetus (e.g., absent or reversed diastolic blood flow in the umbilical artery, pulsation of umbilical vein, or inverted a-wave in the ductus venosus blood flow); and/or (4) fetal hydrops in the pump fetus. Radiofrequency ablation is the least invasive technique. If performed in the first half of pregnancy, the survival rate is around $90 \% .{ }^{11}$

Compared with twin pregnancies with TRAP sequence, the treatment of monochorionic-triplet pregnancies with TRAP sequence is much more challenging. First, it may be technically difficult or even impossible to actually perform an invasive procedure. Second, after the blood flow of the acardiac triplet has been cut off, it could alter the hemodynamics among all three fetuses. Sepulveda et $\mathrm{al}^{10}$ were the first to report that prenatal intervention may not prevent the death of the pump fetus, suggesting that invasive therapy for triplet pregnancy with TRAP sequence may be less effective than for twins. Given the limited number of cases reported thus far, it is difficult to compare the difference between intrauterine treatments and pregnancy outcomes between twins and triplets. Moreover, if the fetal circulations are in series rather than in parallel (i.e., if there is both an indirect and direct pump triplet), as in our case, two fetuses are supporting the blood flow to the acardiac fetus, which may lower their a priori risk of hemodynamic instability leading to fetal hydrops or intrauterine fetal demise. Conservative management without selective reduction may, therefore, have a more favorable outcome in such triplet pregnancies than in twins. The management of monozygotic triplet pregnancies complicated by TRAP sequence should be individualized, but expectant management without invasive intervention can have a favorable outcome.

\section{Ethical Approval}

Guangxi Zhuang Autonomous Region Women and Children Care Hospital Ethics Committee approved the publication of this article.

\section{Acknowledgment}

All authors sincerely thank ShengHe for advice and for revising this article. The authors thank all the people who participated in this study. This study was supported by the Guangxi Science and Technology Project (Gui 1598012-21) and the Health Department of Guangxi Province (Z2016095). The authors declare that there is no competing interest for this case series.

\section{References}

1 Iwamoto H, Yoshida A, Suzuki H, et al. Monochorionic triamniotic triplet pregnancies with assisted reproductive technology: two case reports. J Obstet Gynaecol Res 2010;36(04): 872-875

2 Ghulmiyyah LM, Perloe M, Tucker MJ, Zimmermann JH, Eller DP, Sills ES. Monochorionic-triamniotic triplet pregnancy after intracytoplasmic sperm injection, assisted hatching, and two-embryo transfer: first reported case following IVF. BMC Pregnancy Childbirth 2003;3(01):4

3 Imaizumi Y. A comparative study of zygotic twinning and triplet rates in eight countries, 1972-1999. J Biosoc Sci 2003;35(02): 287-302

4 Argoti PS, Bebbington MW, Johnson A, Moise KJ Jr. Indirect pump: unique presentation of a monochorionic-triamniotic triplet gestation complicated by TRAP sequence and successfully managed with radiofrequency ablation of the acardiac fetus. Ultrasound Obstet Gynecol 2013;42(01):115-117

5 Kirubamani NH. Successful pregnancy in triplet with trap sequence. Indian J Sci Technol 2012;5(09):3304-3306

6 Bianchi DW, Crombleholme TM, D’Alton ME. Fetology: Diagnosis and Management of the Fetal Patient. 2nd ed. New York: McGrawHill Professional; 2010

7 Moore TR, Gale S, Benirschke K. Perinatal outcome of forty-nine pregnancies complicated by acardiac twinning. Am J Obstet Gynecol 1990;163(03):907-912

8 Sepulveda W, Wong AE, Bustos JC, Flores X, Alcalde JL. Acardiac fetus complicating a triplet pregnancy: management and outcome. Prenat Diagn 2009;29(08):794-799

9 Kuran J, Niszczota C, Kolesnik A, Dangel JH. P23.15:Monochorionic triamniotic triplet pregnancy complicated by twin reversed arterial perfusion (TRAP) sequence. Ultrasound Obstet Gynecol 2012;40(Suppl 1):259

10 Søgaard K, Skibsted L, Brocks V. Acardiac twins: pathophysiology, diagnosis, outcome and treatment. Six cases and review of the literature. Fetal Diagn Ther 1999;14(01):53-59

11 Bharadwaj MK, Mohana Priya N. A rare complication of monochorionic twin pregnancy: Twin-reversed arterial perfusion (TRAP) sequence. Med J Armed Forces India 2015;71(Suppl 1): S114-S115

12 López-Pérez R, Lorente M, Martínez-Uriarte J, et al. Twin- reversed arterial perfusion sequence in a triple monochorionic pregnancy with two direct pump fetuses results in significant cyclic Doppler waveform. Fetal Diagn Ther 2015;37(02):157-160 